\title{
Câncer colorretal: principais complicações e a importância do diagnóstico precoce
}

\author{
Colorectal cancer: main complications and the importance of early detection
}

Cáncer colorrectal: principales complicaciones y la importancia del diagnóstico precoz

Sarah Menezes Gashti ${ }^{1 *}$, André Luiz Caramori Tondo², Isabella Freitag ${ }^{3}$, Juliana Maria Mendes de Araújo ${ }^{4}$, Liliane Rochemback ${ }^{5}$, Luiza Orth ${ }^{3}$, Marina Lira ${ }^{3}$, Paula Donizete Rezende ${ }^{6}$, Silvia Campos Gomes ${ }^{1}$, Helen Brambila Jorge Pareja ${ }^{7}$.

\section{RESUMO}

Objetivo: Revisar a literatura com o objetivo de identificar as principais complicações do câncer colorretal (CCR). Revisão Bibliográfica: O CCR é um tumor maligno responsável por ser a terceira causa de morte associada ao câncer no Brasil. O CCR pode ser classificado como familiar ou esporádico, sendo o último, o mais predominante. Devido ao diagnóstico tardio, por um déficit no rastreio adequado, há o aumento de incidência de complicações, sendo elas: obstrução intestinal, a qual corresponde a $24 \%$ de prevalência nos estágios avançados da doença; perfuração intestinal, tem incidência que pode variar entre 2,6\% a 12\% e, dessa forma, é considerada a mais letal por estar relacionada às peritonites secundárias; e hemorragia colorretal, sendo comum, atingindo $50 \%$ dos pacientes. Considerações Finais: Evidencia-se a importância do rastreamento precoce do CCR, a fim de minimizar complicações decorrentes do diagnóstico tardio e, com isso, atenuar agravos no prognóstico do paciente, proporcionando a cura.

Palavras-chave: Câncer colorretal, Perfuração intestinal, Obstrução intestinal.

\begin{abstract}
Objective: To review the literature with the aim of identifying the main complications resulting from colorectal cancer (CRC). Bibliographic review: $C R C$ is a malignant tumor responsible for being the third leading cause of death associated with cancer in the world and in Brazil. CCR can be classified as hereditary or sporadic, the second one is the most prevalent. Due to a delayed diagnosis, there is an increased incidence of complications, such as: bowel obstruction, which reaches $24 \%$ prevalence in advanced stages of the disease; intestinal perforation as an incidence that can vary between $2.6 \%$ to $12 \%$, and is also considered the most lethal because it is related to secondary peritonitis potentially lethal; and colorectal hemorrhage, which is a frequent complication that affect $50 \%$ of patients. Final Considerations: Point the importance of early CRC screening, in order to mitigate complications resulting from late identification and, in consequence, attenuate aggravations in the patient's prognosis.
\end{abstract}

Keywords: Colorectal cancer, Intestinal perforation, Intestinal obstruction.

\footnotetext{
${ }^{1}$ Universidade do Planalto Central Apparecido dos Santos (UNICEPLAC), Brasília - DF.

*E-mail: sarahgashti@hotmail.com

${ }^{2}$ Centro Universitário Integrado (CEI), Campo Mourão - PR.

${ }^{3}$ Centro Universitário da Fundação Assis Gurgacz (FAG), Cascavel - PR.

${ }^{4}$ Faculdade de Medicina de Barbacena (FAME/ FUNJOBE), Barbacena - MG.

${ }^{5}$ Faculdade Estácio de Jaraguá do Sul, Jaraguá do Sul - SC.

${ }^{6}$ Centro Universitário Municipal de Franca (Uni-FACEF), Franca - SP.

7 Universidade do Oeste Paulista (Unoeste), Guarujá - SP
} 


\section{RESUMEN}

Objetivo: Revisar la literatura para identificar las principales complicaciones derivadas del cáncer colorrectal. Revisión bibliográfica: EI CCR es un tumor maligno responsable de ser la tercera causa de muerte asociada al cáncer en todo el mundo y en Brasil. La CCR puede clasificarse en familiar o esporádica, siendo esta última la más prevalente. Debido a un diagnóstico tardío, y debido a un déficit en el cribado adecuado, se incrementa la incidencia de complicaciones, a saber: obstrucción intestinal, a cuál corresponde al $24 \%$ de la prevalencia en los estadios avanzados de la enfermedad; perforación intestinal, tiene una incidencia que puede variar entre el $2,6 \%$ y el $12 \%$, y también es considerada la más letal por estar relacionada con las peritonitis secundarias; y hemorragia colorrectal, que es común y afecta al 50\% de los pacientes. Consideraciones finales: Se destaca la importancia de la detección precoz de CCR para mitigar las complicaciones derivadas de la identificación tardía y, por tanto, atenuar los agravamientos en el pronóstico del paciente.

Palabras clave: Cáncer colorrectal, Perforación intestinal, Obstrucción intestinal.

\section{INTRODUÇÃO}

O Câncer Colorretal (CCR) abrange uma gama de tumores malignos que afetam o intestino grosso e o reto. Assim, podem se manifestar em qualquer extensão do órgão: em maior quantidade (51\%) no cólon sigmóide, cólon ascendente (16\%), cólon descendente (10\%), cólon transverso (9\%), ceco (8\%) e reto (6\%). O CCR, segue um padrão de desenvolvimento, no qual inicialmente mostra-se como uma lesão benigna que evolui para uma neoplasia maligna. Nessa realidade, estima-se que este processo ocorra em torno de 10 a 15 anos (SOUZA RHS, et al., 2016; DA SILVA M e ERRANTE PR, 2017; DE MENEZES CCS, et al., 2016; RAMOS RF, et al., 2017).

As causas do CCR podem ser classificadas como familiar ou esporádica. O primeiro ocorre devido a fatores genéticos que podem ser associados a fatores ambientais. Além disso, é ratificado que as chances para o seu desenvolvimento aumentam em indivíduos com parentes de primeiro grau diagnosticados com essa patologia. Já o esporádico representa a maioria dos casos e vincula-se ao acúmulo de mutações vitalícias (KAYA S, et al., 2019; PACHECO-PÉREZ LA, et al., 2019; DA SILVA M e ERRANTE PR, 2017; MELLO MRSP, et al., 2020).

Apesar da diminuição da incidência mundial de CCR em 20\%, nos últimos 20 anos, este permanece como uma das principais causas de mortalidade mundial. De acordo com o Instituto Nacional de Câncer (INCA), no Brasil em 2018, essa representou, entre os casos de neoplasia, a terceira maior causa de óbito. Ademais, ressalta-se que o CCR possui um alto índice de diagnóstico tardio, o que pode ser explicado em decorrência do estado assintomático do indivíduo ou por apresentar manifestações clínicas inespecíficas (DECKER KM, et al., 2020; KAYA S, et al., 2019; SIMON K, 2016).

Nesse cenário, é necessário ratificar que o prognóstico desse câncer se relaciona com a existência de agravos no processo saúde-doença do paciente. De tal forma, pode-se elencar as principais complicações: obstrução intestinal, perfuração de cólon, sangramento gastrointestinal, anemia, emagrecimento, fístula e intussuscepção. Sendo que dentre essas, nas admissões de emergência, a obstrução e a perfuração são as apresentações clínicas mais presentes, representando 19\% e $8 \%$ das ocorrências respectivamente. Em tal realidade, ressalta-se que essas são as responsáveis por até 30\% dos casos de admissão hospitalar, haja vista que se associam a estágios avançados da patologia (RAMOS RF, et al., 2017; DECKER KM, et al., 2020).

Destarte, estima-se que de 10 a $19 \%$ dos pacientes com neoplasia colorretal desenvolvem tais complicações, o que constitui fator de risco para aumento dos índices de morbidade e mortalidade nessa população. Nesse panorama, observa-se que a história natural da doença apresenta um longo período préclínico. Assim, fica evidente a importância do rastreamento e diagnóstico precoce como estratégias de diminuição de agravos. Sendo que o rastreio é recomendável a partir de 50 anos para pacientes sem histórico familiar de CCR, enquanto para aqueles com casos familiares orienta-se o início de tal estratégia com 40 anos ou 10 anos antes da idade do acometimento do membro da família mais jovem (DE MENEZES CCS, et al., 2016; SOUZA RHS, et al., 2016; BUSTAMENTE-LOPEZ LA, et al., 2019). 
Diante disso, o objetivo desta revisão consiste em evidenciar as principais complicações do CCR. Tendo em vista que essas, quando diagnosticadas precocemente, possibilitam um melhor prognóstico para 0 paciente. Portanto, busca-se enfatizar tais agravos para profissionais da área da saúde, salientando a gravidade da existência desses no processo saúde-doença dos enfermos.

\section{REVISÃO BIBLIOGRÁFICA}

O CCR é considerado a neoplasia de maior frequência do trato digestivo, contemplando 9 a $10 \%$ de todos os cânceres no mundo. Possui grande incidência em países em desenvolvimento, sendo considerado o terceiro câncer que mais afeta homens e o segundo que mais atinge mulheres. Ao lado do câncer de mama e colo do útero, o câncer colorretal possui uma política de rastreamento recomendada pelo Ministério da Saúde, a qual inclui a pesquisa de sangue oculto nas fezes, recomendada para indivíduos a partir dos 50 anos. O índice de mortalidade do CCR em fases precoces da doença atinge cerca de 8,5\% dos acometidos (MORENO DPV, et al., 2020; KAYA S, et al., 2019; RAMOS RF, et al., 2017; SANGUINETTI JM, et al., 2017).

As causas do CCR são classificadas como esporádica ou familiar, a esporádica é predominante, cerca de $75 \%$ dos casos, devido a inúmeras mutações que ocorrem no decorrer da vida do paciente, enquanto que 0 CCR familiar atinge 20 a $25 \%$ dos indivíduos acometidos pela neoplasia - fato atrelado, sobretudo, à mutação do gene APC. Quanto ao hereditário, uma das causas que o gera é a Síndrome de Lynch (SL). Em pessoas com predisposição em desenvolver essa síndrome, os cuidados com os fatores ambientais, como tabagismo e alcoolismo, devem ser redobrados, já que nessa população há $5 \%$ de chance de desenvolver o CCR precoce. Além disso, inúmeros estudos mostram que uma dieta inadequada associada ao sedentarismo contribui no desenvolvimento desse câncer (KAYA S, et al., 2019; SIMON K, 2016; MELLO MRSP, et al., 2020; PACHECO-PÉREZ LA, et al., 2019; MORENO DPV, et al., 2020).

No Brasil, comumente o diagnóstico de CCR é feito já nas fases avançadas da doença, em cerca de $55 \%$ a $70 \%$ dos pacientes. Assim, quanto mais tarde for feito o diagnóstico, maior é a probabilidade de complicações e chances de um prognóstico reservado, com abordagens terapêuticas mais agressivas ou apenas paliativas (SOUZA RHS, et al., 2016).

A mortalidade vinculada ao carcinoma colorretal sofre impacto de um conjunto de fatores, sejam eles modificáveis ou não modificáveis, dos quais se pode citar: alimentação rica em carnes vermelhas e processadas, gorduras, baixo consumo de fibras, sedentarismo, sobrepeso, tabagismo, alcoolismo, diabetes, obesidade e idade avançada - acima de 50 anos, exceto em portadores da Síndrome de Lynch, nos quais 0 CCR pode se desenvolver antes dos 30 anos (DE MENEZES CCS, et al., 2016; PACHECO-PÉREZ LA, et al., 2019).

Por acometer em especial idosos, esse câncer é mais comum em pessoas com mais de 50 anos, logo, o seu rastreamento é importante, pois permite a identificação inicial do câncer, o qual ainda pode ser assintomático (RAMOS RF, et al., 2017; MELLO MRSP, et al., 2020).

\section{Sintomatologia do CCR}

A apresentação clínica é, usualmente, livre de sintomas. Todavia, alguns sintomas podem ser evidenciados e, indubitavelmente, deve-se ter vigilância quanto a sua existência, por exemplo, mudanças no padrão intestinal e nas fezes, dor abdominal e sangue oculto. O perfil sintomatológico pode revelar-se multifacetado, a depender do seu tipo e localização, e englobar uma pluralidade de manifestações - dispneia, cansaço e insônia -, além dos sintomas comumente atrelados à neoplasia intestinal, como náusea, inapetência, dor abdominal, constipação e diarreia. Sintomas pouco prevalentes, como fezes associadas a muco, anemia, dor infra-abdominal, massa abdominal palpável, oclusão intestinal súbita, fístulas no cólon e perfuração intestinal que evolui para peritonite fecal também devem ser valorizados (DE MENEZES CCS, et al., 2016; MOURA SF, et al., 2020).

Os sintomas variam de acordo com a localização tumoral. Nesse sentido, sabe-se que a perda sanguínea pelas fezes pode revelar-se por meio de um sangramento vivo, indicando um tumor localizado em regiões mais distais do intestino. Quando localizado no reto, é frequente sangramentos, que podem se misturar com 
fezes, muco e pus. Contudo, quando associado às fezes escurecidas (melena), tem-se como provável origem o cólon proximal. Ainda, o câncer que acomete o lado direito do cólon possui como sintomas diarreia, anemia, massa palpável no cólon e dor abdominal em especial na fossa ilíaca direita (BUSTAMANTE-LOPEZ LA, et al., 2019; MOURA SF, et al., 2020; SILVA M, et al., 2016).

Ademais, notável discriminação deve ser estabelecida ao se comparar diferentes faixas etárias: enquanto idosos exibem prevalentemente sintomas atrelados à natureza psíquica (como tristeza), os pacientes jovens exteriorizam, em sua maioria, sintomas físicos (como dor). Essa conjuntura fomenta o desenvolvimento de abordagens diferenciadas e individualizadas para cada paciente, a depender de sua idade e em concordância às suas principais queixas (MOURA SF, et al., 2020; SILVA M, et al., 2016).

\section{Rastreio e diagnóstico}

O rastreamento do CCR pode ser fracionado de acordo com o risco dos pacientes: aqueles que exibem baixo risco de desenvolver a malignidade podem ser investigados por meio de três procedimentos: (1) pesquisa de sangue oculto nas fezes, realizada a cada ano - porém em alguns casos, o resultado pode ser impreciso, sendo reflexo de perda de sangue por doenças diverticulares, neoplásicas, hemorroidas ou até mesmo doenças inflamatórias; (2) sigmoidoscopia flexível a cada cinco anos; ou (3) retossigmoidoscopia a cada dois anos para os pacientes com mais de 50 anos de idade (DA SILVA M e ERRANTE PR, 2017).

No que concerne ainda sobre rastreamento, para indivíduos que possuem alto risco de manifestar o câncer, sugere-se que, a partir de 40 anos, o rastreio seja feito por intermédio de colonoscopia, pois permite detalhar as bordas da lesão, seu grau de penetração e também sua superfície. Este exame permite, inclusive, a realização de biópsia para posterior confirmação do diagnóstico e, em seguida, o estadiamento pode ser concluído. Para tal, podem ser úteis tomografias computadorizadas de tórax, pelve e abdômen, além do exame físico e quantificação do antígeno carcinoembrionário (DE MENEZES CCS, et al., 2016).

De acordo com a Sociedade Brasileira de Cancerologia (SBC), para o grupo de alto risco (que compreende indivíduos com história familiar de câncer colorretal), o rastreamento deve ser iniciado entre 35 e 40 anos de idade por meio da pesquisa de sangue oculto nas fezes e um exame endoscópico retal, além da retossigmoidoscopia que deve ser realizada a cada 3 a 5 anos (SOFFEL EM, et al., 2015; PISANO M, et al., 2018)

O grupo de risco normal, tem indicação de rastreio, conforme o que preconiza a SBC, por meio da realização anual da pesquisa de sangue oculto nas fezes e sigmoidoscopia a cada 3 a 5 anos. Entretanto, a Sociedade Americana de Oncologia Clínica (ASCO) recomenda rastreio para o CCR em pacientes assintomáticos com idades entre 50 e 75 anos e risco médio de câncer, em locais onde a incidência dessa neoplasia é alta. Assim, esse processo deve ser feito por meio de pesquisa de sangue oculto nas fezes a cada um ou dois anos. Para o grupo que possui síndromes familiares, o rastreamento deve ser iniciado precocemente (LOPES G, et al, 2019).

Com relação ao método diagnóstico de eleição para obstrução e perfuração intestinal, tem-se que a tomografia computadorizada (TC), por sua alta sensibilidade e especificidade, desempenha essa função de modo mais eficaz quando comparada às demais técnicas, como ultrassonografia (US) e raio X, considerados exames de triagem. Desse modo, é possível inferir que a confirmação diagnóstica deve ser precedida por um destes exames de triagem, para que, em seguida, a TC possa conceder informações singulares e minuciosas acerca das complicações (PISANO M, et al., 2018).

\section{Obstrução}

A obstrução intestinal maligna é hegemonicamente considerada a principal complicação do câncer colorretal, abrangendo $24 \%$ de prevalência nos estágios avançados da doença. Essa complicação é definida como um indicativo clínico de obstrução intestinal distal ao ligamento de Treitz relacionada à proliferação anormal intra-abdominal de células de um tecido primário ou de um câncer extra-abdominal com disseminação pelo peritônio (SOUSA JHB, et al., 2019).

Dentre os mecanismos que propiciam o desenvolvimento de um quadro obstrutivo a nível intestinal, destaca-se tanto a invasão do plexo nervoso quanto a invasão mural da parede muscular, ambas repercutem 
em desordens de motilidade. Não somente a obliteração mecânica provocada pelo tumor, mas a mobilidade intestinal também é atenuada em função de substâncias neuroendócrinas liberadas que ocasionam, inclusive, edema da mucosa e contribuem para elevação da pressão a nível intestinal e consequente oclusão vascular com risco de sepse (LEITE MLLF e BARBOSA LER, 2019).

A divergência no diâmetro luminal entre os lados direito e esquerdo do cólon elucida a predominante ocorrência de obstrução no sigmoide, pois o cólon esquerdo é mais estreito e a consistência fecal é mais densa (quando comparada ao aspecto das fezes do cólon direito). Além disso, a sintomatologia manifestada pelos pacientes acometidos pela obstrução intestinal maligna é bastante diversificada: dor abdominal em cólica, distensão, vômitos, alteração na apresentação das fezes e flatulências. No que concerne à configuração clínica, a própria obstrução pode ser o primeiro sintoma do carcinoma colorretal manifestado em $8 \%$ a $29 \%$ dos pacientes e tal situação acomete $10 \%$ a $19 \%$ dos pacientes (BAER C, et al., 2017; LEITE MLLF e BARBOSA LER, 2019; RAMOS RF, et al., 2017).

A oclusão intestinal provocada pela impactação tumoral no intestino grosso encontra-se, frequentemente, associada à cessação da eliminação de flatulências e fezes, as quais podem ser acompanhadas ou não de sangue, além de distensão abdominal. Esse quadro pode conduzir a achados peculiares no exame físico, como sensibilidade à palpação e ausculta hipercinética ou até mesmo silenciosa em virtude da circunstância em que se encontram os ruídos intestinais. Quando a obstrução do cólon está atrelada a vômitos, mesmo sendo um quadro menos usual, avaliações quantitativas laboratoriais podem ser úteis para determinar a presença de alcalose metabólica (PISANO M, et al., 2018).

Em casos de dilatação intestinal severa, as funções respiratórias e circulatórias também podem ser comprometidas. A perda de fluídos e eletrólitos pode ser observada na obstrução intestinal baixa, já que há a diminuição da absorção e o aumento das secreções da mucosa intestinal, levando a uma grande retenção de fluidos, que são encerrados no intestino e assim impedidos de entrar no sangue. Também, se a obstrução for estrangulada, esses fluidos retidos podem ter elevados números de bactérias, sangue e tecidos necróticos, levando à infecção (YANG XF e PAN K, 2014).

O tratamento consiste em correção do desequilíbrio ácido-base; por meio da sonda nasogástrica é feita a descompressão gastrointestinal; controle de infecção através de terapias com antibióticos de amplo espectro; cirurgia de remoção da obstrução e restauração da função intestinal, a fim de evitar necrose e perfuração. Para o câncer de cólon direito acompanhado de obstrução aguda, opta-se pela hemicolectomia direita com anastomose primária enquanto a ressecção primária do tumor é preferida para a obstrução aguda causada pelo tumor de colón esquerdo (YANG XF e PAN K, 2014).

\section{Perfuração}

Esta é a segunda principal complicação provocada pelo CCR, a qual além de ter uma incidência de 2,6\% a $12 \%$ também é considerada a mais letal por estar relacionada às peritonites secundárias que evoluem para sepse e óbito. A localização predominante da perfuração intestinal se dá no ceco em virtude da sua espessura luminal. O estiramento da parte inicial do intestino grosso faz com que se eleve sua pressão intraluminal, implicando em possíveis complicações. Apesar de existir a possibilidade de perfuração em estágio inicial, o ceco é considerado como fator de risco quando seu diâmetro atinge $12 \mathrm{~cm}$ (BAER C, et al., 2017; JAFFE T e THOMPSON WM, 2015; MADDU KK, et al., 2014; MOON JY, et al., 2019; RAMOS RF, et al., 2017).

A taxa de mortalidade associada à perfuração intestinal varia conforme amplitude: se contida, essa oscila entre $0 \%$ e $24 \%$, ao passo que, se difusa, coexistindo peritonite fecal, os índices atingem $19 \%$ a $65 \%$. Em geral, quadro de perfuração colorretal é tido como uma emergência cirúrgica, pois pode evoluir com choque séptico, coagulação intravascular disseminada, falência de órgãos e, por fim, a morte. Assim, a cirurgia visa à lavagem do local somada à identificação da perfuração. Deve-se, mesmo sem a confirmação do diagnóstico de malignidade, realizar ressecção oncológica associada à linfadenectomia. A conduta se altera conforme o local do tumor: se for no lado direito, pode ser realizada hemicolectomia direita seguida de anastomose primária; caso no lado esquerdo, remoção do tumor e colostomia proximal (PISANO M, et al., 2018; BAER C, et al., 2017; YANG XF e PAN K, 2014). 
A gênese da perfuração intestinal em consequência do CCR revela-se por meio de dois mecanismos distintos: necrose tumoral que compreende o local em que o tumor se desenvolve; e distensão do cólon proximal em virtude de uma obstrução provocada pelo crescimento da massa tumoral. Nesse sentido, o prognóstico associado à perfuração proximal é desfavorável quando comparado à perfuração local, uma vez que a peritonite ocasionada por esta última tende a manifestar-se de modo localizado e contido em oposição à disseminação propiciada pela perfuração proximal, a qual pode culminar em choque séptico. Assim, é coerente enfatizar que o tratamento para as situações mencionadas difere. Logo, é feita a ressecção acompanhada ou não de anastomose e estoma no caso de perfuração no local do tumor. Já para as perfurações proximais, indica-se ressecção tumoral paralela (OTANI K, et al., 2018; PISANO M, et al., 2018).

\section{Hemorragia colorretal}

Complicação muito comum em pacientes com CCR, atingindo até $50 \%$ deles, contudo, não necessita de intervenção cirúrgica de emergência, pois costuma ser de baixo volume e autolimitada. Sintomatologicamente, o paciente com sangramento pode cursar com anemia, em razão do déficit de plasma causado após sangramento maciço. Em uma tentativa de compensação, há importante migração de fluidos teciduais para os vasos sanguíneos, causando queda no nível de hemoglobina e no hematócrito devido à diluição (BAER C, et al., 2017).

Ainda na conjuntura sintomatológica, outra manifestação típica de sangramento de tumor de cólon é o sangue nas fezes, sendo que o sangramento do cólon direito se manifesta com fezes marrons, enquanto $o$ sangramento colônico esquerdo com fezes vermelhas brilhantes. Esses pacientes também podem apresentar circulação periférica hemorrágica ineficiente. Exames podem ser solicitados para auxílio da caracterização de sangramento, dentre eles pode-se citar a aspiração por sonda nasogástrica e endoscopia, cuja eficácia é alta para identificar a origem do sangramento (YANG XF e PAN K, 2014; BAER C, et al., 2017).

$\mathrm{O}$ tratamento não cirúrgico inclui acesso intravenoso adequado; suplementação com cristaloides e coloides para correção do choque; transfusão de sangue de acordo com volume perdido e agentes hemostáticos. Ainda assim, caso haja insucesso no tratamento não cirúrgico, opta-se pelo tratamento cirúrgico, em que é feita a cirurgia de emergência ou cirurgia eletiva dependendo das condições da doença, sendo a forma mais efetiva de conter a hemorragia colorretal (YANG XF e PAN K, 2014).

\section{o CCR na emergência}

Na maioria dos casos de CCR, a necessidade de cirurgia de emergência está associada a um prognóstico ruim. A taxa de casos que requerem essa cirurgia ainda é alta, entre $7 \%$ e $40 \%$, e com taxas de morbidade e mortalidade de $15 \%$ a $50 \%$ e $6 \%$ a $15 \%$, respectivamente (KAYA S, et al., 2019).

Um estudo retrospectivo (2017) realizado no Hospital Federal de Bonsucesso possibilitou uma análise epidemiológica dos pacientes com câncer colorretal admitidos na emergência e evidenciou que as principais complicações constatadas por essa pesquisa foram obstrução $(78 \%)$ e perfuração intestinal $(15 \%)$, as quais revelaram similares taxas de sobrevida. Com relação à localização dos carcinomas colorretais analisados por esse estudo, o predomínio se deu no cólon sigmoide, contemplando $51 \%$ dos casos, enquanto que o reto compreendeu apenas 6\% (RAMOS RF, et al., 2017).

Além da obstrução e perfuração intestinal, verificou-se que dor, emagrecimento, anemia, sangramento, fístula e intussuscepção também fazem parte da parcela de sintomas evidenciados em tais serviços. Ademais, fígado, peritônio, útero e anexos, parede abdominal e pulmão compreendem os sítios de metástases mais frequentemente acometidos pelo CCR sendo que, no estudo retrospectivo previamente mencionado, $42 \%$ dos pacientes apresentavam tal disseminação tumoral (RAMOS RF, et al., 2017).

Em um estudo realizado por Decker KM, et al. (2020), foi analisado que, em virtude do quadro clínico em que se encontra o paciente com admissão hospitalar de emergência, este consequentemente possui um pior prognóstico quando comparado ao paciente com CCR não emergencial e geralmente fica internado o dobro de dias em relação ao paciente não emergencial, gerando alto custo para o hospital que o admitiu.

Avaliou-se 179 pacientes com adenocarcinoma que necessitavam de tratamento emergencial devido a peritonite, achados de sepse abdominal ou sistêmica e obstrução. Destes, 152 apresentavam obstrução 
completa e 27 perfurações. Em relação à posição anatômica do tumor, pacientes com câncer no cólon esquerdo apresentavam mais obstrução e perfuração. Concluiu-se nesse estudo que as principais complicações foram morbidades relacionadas aos sistemas gastrointestinal, respiratório, circulatório e excretor (KAYA S, et al., 2019).

\section{CONSIDERAÇÕES FINAIS}

Verificou-se nesse trabalho a relevância do câncer colorretal (CCR) e as suas principais complicações (obstrução, perfuração e hemorragia). Destarte, constatou-se que esse tipo de câncer segue um padrão de desenvolvimento, inicia-se como uma lesão benigna e evolui para uma neoplasia maligna. Durante o estudo foi visto que é uma das principais causas de morte no mundo, acomete predominantemente a população idosa. Em função disso, explica-se a importância do rastreamento, a qual permite a identificação inicial do câncer, quando este ainda é oligossintomático ou assintomático.

\section{REFERÊNCIAS}

1. ALATISE OI, et al. A symptom-based model to predict colorectal cancer in low-resource countries: Results from a prospective study of patients at high risk for colorectal cancer. Cancer, 2018; 124(13): 2766-2773.

2. BAER C, et al. Apresentações de emergência de câncer colorretal. Clínicas Cirúrgicas, 2017; 97(3): 529-545.

3. BICUDO-SALOMÃO A, et al. Fatores associados à redução do risco de complicações na cirurgia colorretal com cuidados pré-operatórios recomendados pelo projeto acerto. ABCD. Arquivos Brasileiros de Cirurgia Digestiva (São Paulo), 2019; 32(4).

4. BUSTAMANTE-LOPEZ LA, et al. Existe diferença entre câncer do cólon direito versus o esquerdo? A localização faz alguma diferença no seguimento em longo prazo? ABCD. Arquivos Brasileiros de Cirurgia Digestiva (São Paulo), 2019; 32(4).

5. DA SILVA M, ERRANTE PR. Câncer colorretal: fatores de risco, diagnóstico e tratamento. UNILUS Ensino e Pesquisa, 2017; 13(33): 133-140.

6. DE ALBUQUERQUE GOMES AMB, et al. Results of surgical treatment of colorectal cancer in nonagenarian patients. Journal of Coloproctology, 2017; 37(4): 285-289.

7. DE MENEZES CCS, et al. Câncer colorretal na população brasileira: taxa de mortalidade no período de 2005-2015. Revista Brasileira em Promoção da Saúde, 2016; 29(2): 172-179.

8. DECKER KM, et al. Time Trends in the Diagnosis of Colorectal Cancer With Obstruction, Perforation, and Emergency Admission After the Introduction of Population-Based Organized Screening. JAMA network open, 2020; 3(5): e205741e205741.

9. DOS SANTOS AC, et al. Emergency surgery for complicated colorectal cancer in central Brazil. Journal of Coloproctology, 2014; 34(2): 104-108.

10. FERNANDES D, et al. Acute treatment of malignant colorectal occlusion: real life practice. GE Portuguese journal of gastroenterology, 2016; 23(2): 66-75.

11. FRANKE AJ, et al. Management of malignant bowel obstruction associated with GI cancers. Journal of oncology practice, $2017 ; 13(7): 426-434$.

12. JAFFE T e THOMPSON WM. Large-bowel obstruction in the adult: classic radiographic and CT findings, etiology, and mimics. Radiology, 2015; 275(3): 651-663.

13. KAYA S, et al. Evaluation of current therapeutic approach to obstructive and perforated colorectal cancers. Turkish Journal of Trauma and Emergency Surgery, 2019; 25(6): 589-596.

14. LEITE MLLF e BARBOSA LER. Endoscopic stent in malignant colonic obstruction: the risk of tumor seeding. Journal of Coloproctology, 2019; 39(4): 357-364.

15. LOPES G, et al. Early detection for colorectal cancer: ASCO resource-stratified guideline. Journal of global oncology, 2019; 5: 1-22.

16. MADDU KK, et al. Colorectal emergencies and related complications: a comprehensive imaging review-imaging of colitis and complications. American Journal of Roentgenology, 2014; 203(6): 1205-1216.

17. MELLO MRSP, et al. Clinical evaluation and pattern of symptoms in colorectal cancer patients. Arquivos de gastroenterologia, 2020; 57(2): 131-136.

18. MOON JY, et al. Differentiation between stercoral perforation and colorectal cancer perforation. Revista da Associação Médica Brasileira,2019; 65(2): 191-197.

19. MORENO DPV, et al. Revisión: Factores asociados a cáncer colorrectal. Revista Médica de Risaralda, 2020; 2(1): 6877.

20. MOURA SF, et al. Padrão Sintomatológico em Pacientes do Câncer Colorretal de acordo com a idade. Revista Brasileira de Cancerologia, 2020; 66(1).

21. OTANI K, et al. Colon cancer with perforation. Surgery today, 2019; 49(1): 15-20.

22. PACHECO-PÉREZ LA, et al. Fatores ambientais e conscientização sobre o câncer colorretal em pessoas com risco familiar. Revista Latino-Americana de Enfermagem,2019; 27: e3195. 
23. PISANO M, et al. 2017 WSES guidelines on colon and rectal cancer emergencies: obstruction and perforation. World journal of emergency surgery, 2018; 13(1): 1-27.

24. RAMOS RF, et al. Cirurgia no câncer de cólon em pacientes operados de emergência. Revista do Colégio Brasileiro de Cirurgiões, 2017; 44(5): 465-470.

25. RASMUSSEN S, et al. Predictive values of colorectal cancer alarm symptoms in the general population: a nationwide cohort study. British journal of cancer, 2019; 120(6): 595-600.

26. SANGUINETTI JM, et al. Colorectal cancer screening: a South American perspective. Revista de Gastroenterología del Perú, 2020; 40(3): 238-245.

27. SIMON K. Colorectal cancer development and advances in screening. Clinical interventions in aging, $2016 ; 11: 967$.

28. SOTOMAYOR LA, et al. Factores predictores de mortalidad en la oclusión intestinal por cáncer colorrectal y anal. Revista Cubana de Cirugía,2020; 59(1).

29. SOUSA JHB, et al. Surgical management of malignant intestinal obstruction: outcome and prognostic factors. Chirurgia, 2019; $114: 343-351$.

30. SOUZA JLCA, et al. Health-related quality of life assessment in patients with rectal cancer treated with curative intent. Arquivos de gastroenterologia, 2018; 55(2): 154-159.

31. SOUZA RHS, et al. COLORECTAL CANCER: factors related to late diagnosis in users of the public health system treated at an Universitary Hospital in Curitiba, Paraná State, Brazil. Arq. Gastroenterol. São Paulo, 2016; 53(2): 68-75.

32. STEVANATO FILHO PR, et al. Surgical complications and metachronous rectal cancer risk in patients with classic familial adenomatous polyposis. Journal of Coloproctology (Rio de Janeiro), 2015; 35(1): 08-13.

33. STOFFEL EM, et al. Hereditary colorectal cancer syndromes: American society of clinical oncology clinical practice guideline endorsement of the familial risk-colorectal cancer: European society for medical oncology clinical practice guidelines. Journal of clinical oncology, 2015; 33(2): 209.

34. YANG XF, PAN K. Diagnosis and management of acute complications in patients with colon cancer: bleeding, obstruction, and perforation. Chinese Journal of Cancer Research, 2014; 26(3): 331. 